\title{
Versatile Retrovirus Vector Systems for Regulated Gene Expression In Vitro and In Vivo
}

\author{
Dirk Lindemann, Edward Patriquin, Sandy Feng, \\ and Richard C. Mulligan \\ Whitehead Institute for Biomedical Research and Department \\ of Biology, Massachusetts Institute of Technology, \\ Cambridge, Massachusetts, U.S.A.
}

\begin{abstract}
Background: Several plasmid DNA-based mammalian expression systems have recently been developed which make it possible to manipulate gene expression via the administration of exogenous agents. In order to extend the application of these systems, we have developed retroviral vectors which allow for the controlled expression of inserted genes both in vitro and in vivo.

Materials and Methods: Two vector strategies which make use of the tetracycline-regulated gene expression system described by Gossen and Bujard were evaluated. In a first strategy, one virus was generated which encoded the tTA or rtTA transactivator gene product, and a second virus was generated in which expression of the gene of interest was dependent upon tetracycline-responsive transcriptional control elements placed either within the viral LTR or within the proviral transcriptional unit. In a second vector strategy, both components of the tet-regulatable system were incorporated into a single proviral genome in such a way that expression of
\end{abstract}

both the transgene and the transactivator gene product were under control of tet-regulatable control elements. Results: Both vector strategies resulted in the ability to regulate the expression of inserted genes. In one single virus configuration, gene expression could be regulated over $100 \times$ and the level of gene expression in the induced state was comparable to or greater than that achieved with standard LTR-based vectors. The use of different deletions in the viral LTR made it possible to generate a number of vectors which provide for a fourfold range of levels of expression of inserted genes in the induced state. Studies in mice with transduced cells demonstrated that gene expression could be induced in vivo by manipulation of tetracycline for at least 48 days.

Conclusions: The availability of highly transmissible, regulatable retroviral vectors should greatly facilitate studies in which it is of interest to manipulate the expression of specific genes in vitro or in vivo.

\section{INTRODUCTION}

Several plasmid based mammalian expression systems have recently been developed which

Dr. Dirk Lindemann's current address: Institute for Virology and Immunology, University of Würzburg, Versbacher Str. 7, 97222 Würzburg, Germany

Dr. Edward Patriquin and Dr. Sandy Feng's current address: Howard Hughes Medical Institute, The Children's Hospital and Department of Genetics, Harvard Medical School, Boston, MA 02115, U.S.A.

Address correspondence and reprint requests to: Dr. Richard C. Mulligan, Howard Hughes Medical Institute, The Children's Hospital and Department of Genetics, Harvard Medical School, 320 Longwood Ave., Boston, MA 02115, U.S.A. Tel: 617-355-8541; Fax: 617-730-0432; e-mail: mulligan@rascal. med.Harvard.edu. make it possible to manipulate gene expression via the administration of exogenous agents (1-4). To date, the system that has been most widely employed makes use of specific control elements of the $E$. coli Tn10 operon (5) to regulate transcription. This system, originally described by Gossen and Bujard (1), relies on the use of two plasmid constructs: (i) an expression construct which encodes a hybrid transactivator gene product (termed either tTA or rtTA) in which the DNA binding domain of the tetracycline repressor (tetR) of Escherichia coli is linked to the transactivation domain of the herpes simplex (HSV) VPl6 gene product, and (ii) a construct in which expression of the desired gene 
product is controlled by a tetracycline-responsive transcriptional control element generated by the fusion of seven copies of tet operator sequences derived from the Tn10 operon (5) to a minimal cytomegalovirus (CMV) immediate early gene promoter (tetOP/CMV) or other minimal promoter sequences. In the original system described by Gossen and Bujard (1), binding of tTA to the regulatory element is inhibited by the presence of tetracycline and transcription from the tet regulatory unit is virtually silent. In the absence of tetracycline, the specific high affinity binding of tTA to the chimeric tet promoter leads to a dramatic increase in transcription from the regulatory unit. More recently, Gossen et al. (2) have also described a modified system which makes use of a mutant transactivator (termed reverse or rtTA) that binds to the regulatory unit only in the presence of certain tetracycline derivatives. This modification may facilitate the in vivo use of the tet regulatory system and lead to the faster induction of gene expression.

The system of Gossen and Bujard necessitates the transfer into cells of both a vector which encodes the relevant transcriptional transactivator gene product(s) and a second construct in which the cDNA of interest has been placed under the control of specific regulatable transcriptional control sequences $(1,2)$. The utility of this regulatable vector system therefore depends both upon the ability to express sufficient amounts of the necessary transactivator(s) and the integration of the regulatable vector construct in such a way that the basal levels of transgene expression are low, yet the induced levels of expression are high. In studies involving cultured cells, these requirements often necessitate the screening of a large number of stable transfectants and are compounded by the need to introduce both constructs into the same cell. Although the tet-regulatable expression system has already been widely employed in studies involving cultured cell lines, the use of the system to regulate gene expression in primary cells, either in vitro or in vivo, has been hampered by the inability to efficiently transduce the relevant target cells without the use of drug selection. For example, studies involving primary cells in vivo have, to date, necessitated the lengthy process of generating transgenic animals and the breeding of strains carrying the expression and transactivator-encoding constructs $(6,7)$.

In order to extend the application of regulated gene expression systems, we have generated highly transmissible retrovirus vectors which encode the relevant elements of the tetracycline-regulated gene expression system described by Gossen and Bujard $(1,2)$. Here, we describe both 'one virus' and 'two virus' strategies which result in the ability to efficiently generate transduced cells which express the gene of interest in a highly regulated fashion. We also show that cells transduced by the vectors demonstrate regulated expression even after their transplantation in vivo.

\section{MATERIALS AND METHODS}

\section{Construction of Retroviral Vectors}

All constructs are based on the SFG retroviral vector developed in our laboratory (8). In the case of each construct, protein coding sequences were introduced between the Nco I and Bam HI sites of the vector in a precise way, such that the AUG sequences encoded by the Nco I site initiate translation of the gene product of interest. SFG $h G H$ contained the human growth hormone (hGH) ORF (9). SFG Luc was constructed by insertion of the luciferase ORF from the pGL-2 plasmid (Promega Corp., Madison, WI). The SFG $t T A$ and $r$ TTA constructs contained the ORF of the transactivator (tTA) from pUHD15-1 (1) and the reverse transactivator (rtTA) from pUHG17-1 (2). SFG NLS-Lac- $Z$ contained the E. coli beta galactosidase gene fused C-terminal to a SV40 nuclear localization signal (10).

Variants of the basic SFG vectors with modifications in the U3 of the 3'LTR have the type of deletion specified by letters and numbers (e.g. $S F G L u c E C 1$ ). A prefix " $t 7$ " in front of the deletion indicates the insertion of 7 tet operator sequences into the deletion site (e.g. SFG Luc t7EC1). A prefix " $t c$ " in front of the gene name indicates the insertion of tet/CMV regulatory element immediately upstream of the gene (e.g. SFG tc $L u c)$. Vectors expressing the tTA or rtTA ORF linked through an EMCV IRES element downstream of the marker gene are labeled SFG tcLuc IT or SFG tcLuc IRT, respectively.

The following deletions and/or insertions in the $\mathrm{U} 3$ region of the $3^{\prime} \mathrm{LTR}$ were made (positions of the first and last nucleotide of the deletion relative to the transcription start of the 3'LTR are indicated): E1 $(-327 /-152), \mathrm{E} 2(-327 /-116)$, E3 $(-327 /-92)$, E4 $(-412 /-152)$, E5 $(412 /-116)$, E6 $(-412 /-92)$, E7 $(-436 /-92)$, E8 $(-436 /-116)$, E9 $(-436 /-152)$, EC1 $(-327 /-63)$, EC2 $(-412 /$ $-63)$, EC3 $(-436 / 63)$, ECT1 $(-327 /-63+-30 /$ $-24)$, ЕСT2 $(-412 /-63+-30 /-24)$, ЕCT2 
$(-412 /-63+-30 /-24)$, ECT3 $(-436 /-63+$ $-30 /-24), \mathrm{t} 7 \mathrm{CM}(-436 /-1+$ inserted tet-CMV minimal promoter).

\section{Cells and Animals}

C2C12 $(11,12)$ and NIH3T3 cells were obtained from American Type Culture Collection, Rockville, MD and ecotropic BOSC23 (13) packaging cells were a gift from W. Pear. $\mathrm{C} 2 \mathrm{C} 12$ and BOSC cells were cultivated in DMEM containing $10 \%$ fetal calf serum (FCS) and NIH3T3 cells were cultured in DMEM containing $10 \%$ calf serum. All media was supplemented with $100 \mathrm{U} / \mathrm{ml}$ penicillin and $100 \mu \mathrm{g} / \mathrm{ml}$ streptomycin. $\mathrm{C} 3 \mathrm{H} / \mathrm{HeJ}$ mice, 3-4 weeks old, were purchased from Jackson Laboratory (Bar Harbor, ME).

\section{Transfections, Infections, Induction of Gene Expression, and Determination of Transduction Efficiencies}

Recombinant retroviral vectors were generated by transient transfection of the ecotropic BOSC23 packaging cell line as described elsewhere (13). Viral supernatants from independent transfections with the same retroviral construct were pooled, filtered $(0.45 \mu)$, and used immediately or stored at $-80^{\circ} \mathrm{C}$ until use. When using the original tTA gene, BOSC23 cells were grown in the presence of $1 \mu \mathrm{g} / \mathrm{ml}$ tetracycline (Sigma, St. Louis, MO).

For infection of NIH3T3 cells, $1 \times 10^{5}$ cells per $6 \mathrm{~cm}$ dish were plated for 24 hours and then incubated for 4 hours with $3 \mathrm{ml}$ of viral supernatant in the presence of $8 \mu \mathrm{g} / \mathrm{ml}$ Polybrene. The following day the transduced cell population was split into two $6 \mathrm{~cm}$ dishes. In the case of vectors employing the tTA gene product, gene expression was induced 24 hours later on one plate by washing two times and further incubation with tetracycline free growth medium while the other plate continued to be incubated in the presence of tetracycline $(1 \mu \mathrm{g} / \mathrm{ml})$. When using the rtTA gene product, expression was induced on one plate through the addition of doxycycline (Sigma, St. Louis, MO) at $1 \mu \mathrm{g} / \mathrm{ml}$ to the medium. After an additional 48 hours incubation cells of both plates were harvested and gene expression was analyzed.

Transduction efficiency was determined by Southern blot analysis of infected cultures as described previously (14). Briefly, equal amounts of genomic DNA from infected cultures were separated by agarose gel electrophoresis after re- striction enzyme digestion, blotted onto Zetaprobe GT membrane (Biorad) and hybridized with insert specific radiolabelled probes. A mouse IgM constant region specific probe was included to standardize the individual samples. Intensity of the different bands was quantitated on a Fuji Phosphorimager (Fuji, Tokyo, Japan) using the MacBas2.2 software system.

\section{Luciferase Assays}

Luciferase activity of transduced cells was essentially performed as described elsewhere (1). Enzymatic activities where normalized for total protein concentration determined by a Bradford assay (Biorad) and expressed as relative light units (rlu) per $\mu \mathrm{g}$ total protein and the copy number of the luciferase gene in the infected cells as determined by Southern blot analysis.

\section{Regulation of hGH Expression In Vitro and In Vivo}

C2C12 rtTA cells were generated by infection of C2C12 with supernatant of BOSC 23 cells transfected with the SFG rtTA vector. Wildtype C2C12 or $\mathrm{C} 2 \mathrm{C} 12$ rtTA cells were infected with various retroviral vectors for constitutive or regulated hGH expression as indicated. Twenty four hours after infection, $1 \times 10^{6}$ transduced cells were plated in parallel into $10 \mathrm{~cm}$ dishes either in normal media or in doxycycline $(1 \mu \mathrm{g} / \mathrm{ml})$ containing growth media. The next day the growth medium was replaced by the appropriate fresh growth medium and 24 hours later the supernatant was harvested and filtrated and hGH concentrations were determined by ELISA (United Biotech Inc., Mountain View, CA). The sensitivity of the assay was $0.3 \mathrm{ng} \mathrm{hGH/ml}$.

For injection into mice, transduced $\mathrm{C} 2 \mathrm{C} 12$ cell populations were trypsinized and washed twice with Hanks Balanced Salt Solution (HBSS) and finally resuspended in HBSS at a concentration of $3 \times 10^{7}$ cells per ml. Groups of 3-6 anaesthetized $\mathrm{C} 3 \mathrm{H} / \mathrm{HeJ}$ mice, 3-5 weeks old, received $3 \times 10^{6}$ transduced cells per mouse through i.m. injection into the tibialis anterior muscle (50 $\mu$ l per muscle).

Doxycycline (Sigma, St. Louis, MO) was dissolved at $2.2 \mathrm{mg} / \mathrm{ml}$ in deionized water supplemented with $5 \%$ sucrose and administered orally through the drinking water. The flasks were wrapped in tin foil to protect the doxycyline against light and were replaced every third day. Mice not on doxycyline treatment received 
deionized water supplemented with $5 \%$ sucrose. Mice were bled at the indicated time points and hGH serum levels were determined with a hGH specific RIA (Nichols Institute, San Juan Capistrano, CA) according to the manufacturer's instructions. The sensitivity of the assay was $0.15 \mathrm{ng} \mathrm{hGH} / \mathrm{ml}$.

\section{RESULTS}

\section{Strategies for Generating Tetracycline- Regulatable Retroviral Vectors}

Two different strategies for incorporating the different components of the original and modified tet-regulated gene expression systems into retroviral vectors were evaluated (Fig. 1). In a first strategy (termed the "two virus strategy"), one virus was generated in order to provide for the constitutive expression of the chimeric transactivator gene products and a second virus was generated for the purposes of transferring a tetresponsive transcriptional cassette carrying the gene of interest. Co-infection with both viruses is therefore necessary in order to obtain regulated gene expression. The rationale for the two virus strategy is to ensure that the signals used for the constitutive expression of the transactivators do not influence the basal (non-induced) level of expression of the transgene. The high efficiency of gene transfer afforded by the SFG/MFG vectors $(8,14,15)$ suggested that it would be possible to efficiently co-introduce both proviral genomes into the same cell.

To generate the transactivator-encoding viruses, the coding sequences for either the tTA or rtTA gene products were introduced into the vector SFG (8), a derivative of the MFG vector $(14,15)$. The MFG/SFG vectors employ the MMLV LTR and MMLV-derived splicing signals for expression of inserted sequences. The resulting viruses were termed SFG tTA and SFG rtTA (Fig. 1). For transfer of the regulatable transcription cassette, two types of constructs were generated. In one configuration, the 3'LTR of SFG was modified so as to incorporate tet-responsive transcriptional control elements, either by replacement of both viral enhancer and promoter elements with the tetOP/CMV control element (SFG Luc t7CM), or by replacement of the viral enhancer or enhancer/CAAT sequences with the multimeric tetOP element, leaving the viral minimal promoter sequences intact (only one enhancer deficient version of these vectors, SFG

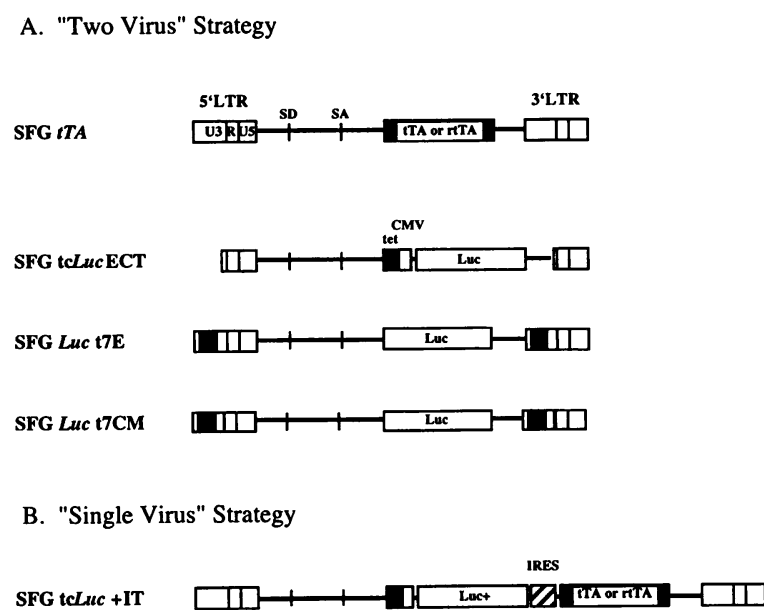

FIG. 1. Schematic map of SFG-based retroviral vectors used in the study

Vectors employed in the "two virus" strategy (A) and "single virus" strategy (B) are shown. For the "two virus" strategy, the vectors include SFG vectors used for constitutive gene expression of the transactivators tTA or rtTA (depicted as SFG tTA), and (i) a retroviral vector in which tetOP/minimal CMV promoter sequences are introduced internal to the proviral transcriptional unit (SFG tc LucECT), (ii) retroviral vectors in which tetracycline response elements (tetOP) are introduced into a $3^{\prime}$ LTR in which the enhancer (or CAAT sequences) are deleted (depicted as SFG Luct7E) (the vector carrying a deletion of enhancer and CAAT sequences, termedSFG Luc7EC, is not shown), or (iii) a retroviral vector in which the tetOP/minimal CMV promoter sequences are introduced in the 3'LTR in place of the viral enhancer/ promoter sequences (SFG Luc t7CM). Also shown is a vector representing the "single virus" strategy (SFG Luc tc LucIT). For all constructs, tTA or rtTA (shaded boxes) represent the original or modified transactivator ORFs; tet (black boxes) represent the tandem repeats of tet operator sequences; CMV (lightly shaded boxes) represents the CMV IE minimal promoter; Luc (open boxes) represents the luciferase ORF; IRES (striped boxes) represents the EMCV internal ribosomal entry site; SD, splice donor; SA, splice acceptor; LTR, long terminal repeat. Details of the construction of the different retroviral vectors are provided in the Materials and Methods section.

Luc t7E, is shown in Fig. 1)(see Materials and Methods for precise description of the deletions). Transmission of the corresponding proviral sequences to cells should result in the representation of the modified transcriptional control sequences in both the $5^{\prime}$ and $3^{\prime}$ LTRs $(16-18)$ and generation of a transgene-encoding transcript identical in structure to that produced by the parental SFG vector. In a second configuration, 
tetOP/minimal CMV promoter sequences were introduced into SFG internal to the proviral transcriptional unit in which the enhancer, CAAT box and TATAAA box were deleted (SFG tcLuc ECT) (Fig. 1). The deletion of sequences in the LTR were introduced in order to decrease or eliminate LTR-mediated transcription after proviral integration (16-18). In both two-virus configurations, the luciferase gene was used as the reporter gene.

In a second strategy (termed the "single virus strategy"), attempts were made to introduce all of the necessary components into a single vector. For the construction of this type of vector, both the tet/CMV promoter sequences and luciferase coding sequences were positioned internal to the proviral transcriptional unit. To provide for expression of the transactivator gene products, picornavirus-derived internal ribosome entry site (IRES) sequences (19) were positioned directly $3^{\prime}$ to the luciferase encoding sequences and $5^{\prime}$ to either the tTA or rtTA coding sequences (SFG tc luc IT) (Fig. 1). With this configuration, we expected that expression of both the transgene and the transactivator would be largely controlled by the tet/CMV promoter sequences, and that low levels of transactivator expression would 'autostimulate' transactivator expression. One likely requirement of such a transcriptional circuit, however, would be that some low level of tetindependent transcription would be needed in order to initiate the autostimulatory circuit. For this reason, we evaluated a number of constructs carrying deletions in the 3'LTR expected to reduce the transcriptional activity of the 5'LTR after proviral integration (see below).

\section{Characterization of the Two-Virus Vector System}

To evaluate the characteristics of the two virus system, NIH 3T3 cells were first infected with either SFG tTA or SFG rtTA virus generated by transient transfection of the BOSC 23 cell line (13) (see Materials and Methods). Southern blot analysis of DNA isolated from cells infected by either virus indicated that virtually all of the cells were infected (data not shown). Next, mockinfected, SFG tTA-, or SFG rtTA infected NIH 3T3 cells were additionally infected with either SFG Luc t7E7, SFG Luc t7EC3, SFG Luc t7CM, or SFG tcLuc ECT3 and subsequently cultured in the presence or absence of tetracycline (in the case of mock- or SFG rtTA infected cells) or doxycycline (in the case of mock- and SFG rtTA infected cells)

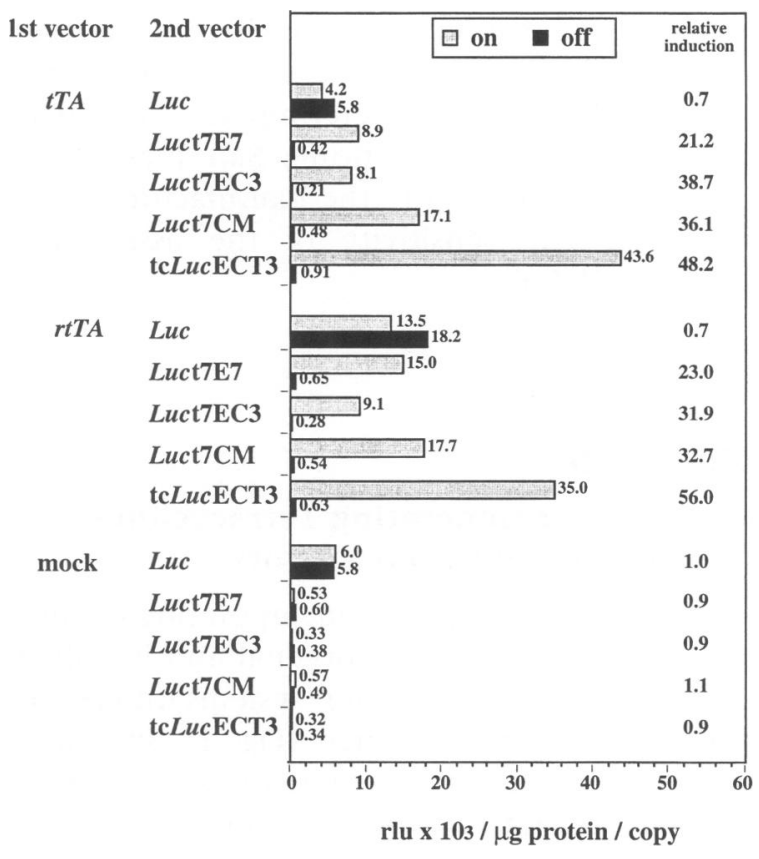

FIG. 2. Regulation of luciferase activity in NIH3T3 cells infected with vectors representing the "two virus" strategy

NIH3T3 cells were first infected with retroviral vectors for constitutive expression of the transactivators tTA, rtTA, or mock incubated with BOSC 23 supernatant alone, as indicated in the "1st vector" column. After splitting into multiple dishes, the three different NIH3T3 populations were superinfected with different vectors, as indicated in the " 2 nd vector" column. The next day the cultures were split into replica plates and gene expression was kept in the off state (solid bar) or turned on (shaded bar) through the addition of doxycycline in the case of rtTA expressing cells or removal of tetracycline in the case of tTA expressing cells. Luciferase activities of cell extracts were determined 48 hours after induction. Enzymatic activities are expressed in relative light units (rlu) and were normalized for the amount of total protein of the samples and the copy number of the luciferase gene in the different cell populations, which was determined by Southern blot analysis. Relative induction levels were calculated by dividing the enzyme activity of the on state through that of the off state from replica plates of the same culture, and are shown in the "relative induction" column.

as described in the Materials and Methods. Extracts were then prepared from the different transduced cells for the determination of luciferase activity (Fig. 2). As expected, in the absence of tTA or rtTA expression, none of the vectors containing tet-regulatable control elements expressed appreciable amounts of luciferase, either in the presence or absence of anti- 
biotic, while SFG Luc infected cells expressed luciferase at high levels, both in the presence and absence of antibiotic. In contrast, in cells transduced by both a transactivator-encoding construct and a tet-regulatable vector, the levels of luciferase expressed could be varied from 20-60 fold, depending on the construct, by the addition or withdrawal of the appropriate antibiotic. In the case of the vectors in which multimerized tet operator sequences were positioned in the viral LTR adjacent to minimal LTR promoter sequences (SFG Luc t7E7 and SFG Luc t7EC3), the deletion of both the enhancer and CAAT sequences in the LTR led to a modest (two-fold), but significant reduction in basal expression, in comparison to deletion of only enhancer sequences without reducing the level of induced expression. Replacement of both the viral enhancer and promoter sequences within the LTR with tetOP/CMV sequences (SFG Luc t7CM) yielded up to two-fold higher levels of induced expression than observed with the SFG Luc t7E7 and SFG Luc t7EC3 constructs, and basal levels comparable to those observed with the SFG Luc t7E7 construct. In the case of the vector configuration in which tet-regulatory sequences were placed internal to the proviral transcriptional unit (SFG tcLuc ECT3), the absolute levels of expression of luciferase in the induced state were at least two-fold greater than any of the other constructs, and the basal levels were comparable to those observed with the SFG Luc 7E7 and SFG t7CM constructs. All of the tet regulatable constructs yielded levels of expression in the induced state which were significantly higher than those observed with the parental SFG vector. In the case of the studies involving SFG tTA transduced cells, the SFG tcLucECT3 vector expressed approximately ten times more luciferase than the SFG Luc vector. Interestingly, both the SFG Luc and the SFG Luct7E7 vectors expressed higher levels of luciferase in rtTA expressing cells.

\section{Characterization of the Single Virus System}

Because it was likely that the efficacy of the single virus system would depend critically upon achieving specific levels of LTR-driven transcription which would be sufficient to initiate tTA expression, yet not lead to a unsuitably high level of transgene expression in the uninduced state, we first directly examined the effects of specific deletions of the LTR on viral transcription in order to select a series of mutations for evaluation in single virus vector constructs. For this study, we generated a number of derivatives of SFG Luc carrying different deletions in the 3'LTR and examined the expression of luciferase in NIH 3 T3 cells 48 hours after infection with virus generated by transient transfection of BOSC 23 cells. As shown in Figure 3A, deletion of increasing amounts of sequence in the LTR led to progressive decreases in luciferase activity. For example, deletion of the core enhancer sequences of the LTR (termed E1) led to an 18-fold decrease in luciferase activity relative to the intact LTR, while the most extensive enhancer deletion (E7) resulted in a 90 -fold decrease in activity. Additional removal of the CAAT box and the TATA box resulted in a further 1.5-5-fold decrease in enzyme activity compared to the enhancer only deletions. Cells infected with the vector having the most extensive deletion of the LTR (ECT3) showed a luciferase activity that was 175-fold below that of the unmodified vector. Southern blot analysis of infected NIH/3T3 cells indicated that all of the viruses faithfully transferred the proviral sequence at high efficiency (approximately 2-3 copies/cell); (data not shown), thereby indicating that the deletions had little or no effect on viral replication/integration. In addition, analysis of luciferase activity in transfected BOSC cells indicated that none of the deletions in the 3'LTR appreciably affected the translational efficiency of the resulting RNAs (data not shown).

Based on the above analysis, single virus constructs carrying either an intact 3'LTR or LTRs carrying the E1, E4, E7, or ECT3 deletions were constructed and virus derived from the constructs was used to infect NIH $3 \mathrm{~T} 3$ cells in order to determine the capacity of the constructs for tet-regulated expression. The results of such an analysis are shown in Figure 3B. Gene expression in cells infected with the SFG tcLuc IT vector, containing an intact MuLV enhancer/promoter, could be stimulated 8-fold in the on state. However, in the off state, luciferase activity was only slightly below that of the constitutively expressing SFG Luc vector, which was not regulatable by tetracycline treatment. A dramatic increase in the relative regulation level of luciferase activity was observed with the vectors harboring various deletions in the MuLV LTR. Progressive deletion of viral enhancer/promoter sequences led to a progressive decrease in the basal level of luciferase expression. Interestingly, however, the decrease in basal expression associated with the increasing deletion of LTR se- 


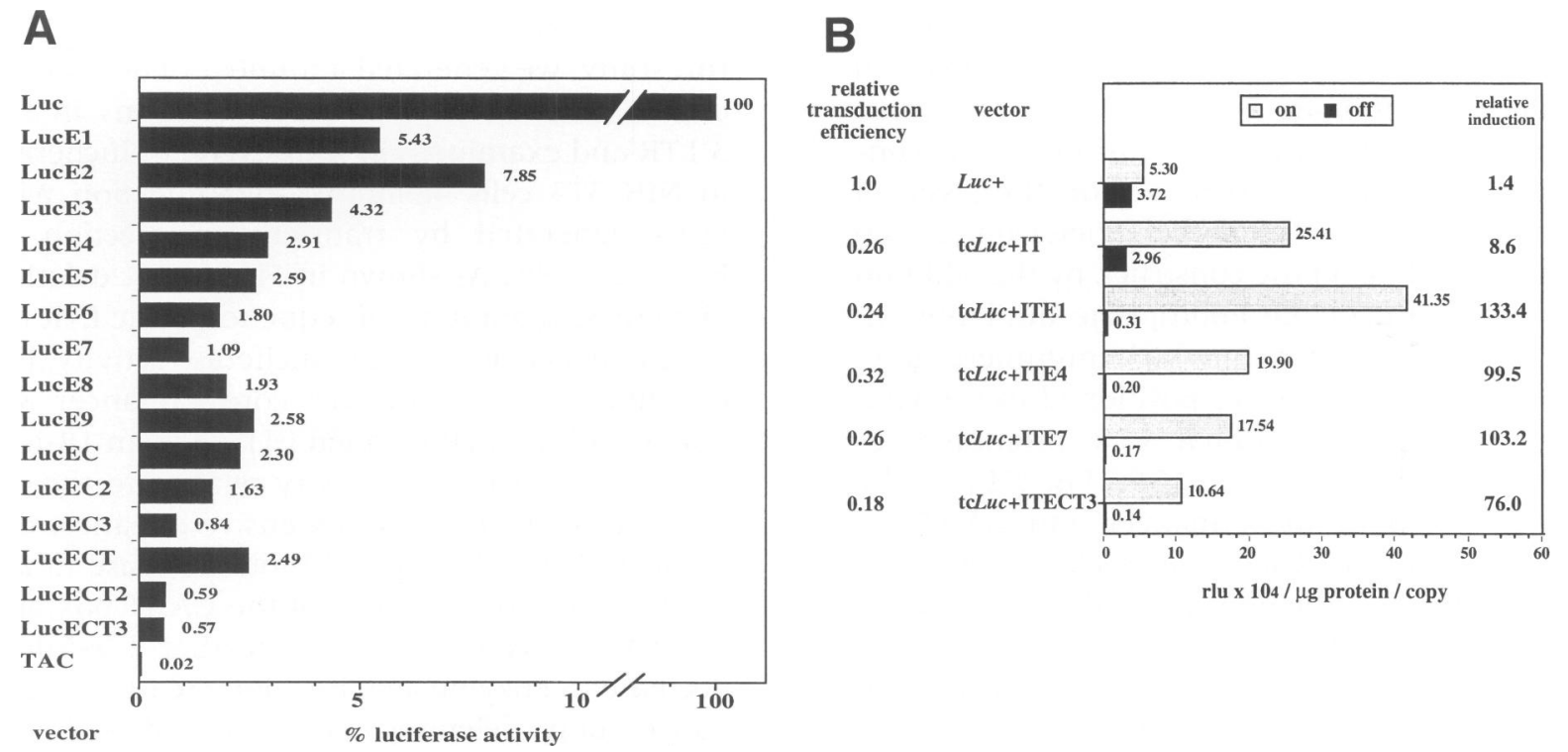

FIG. 3. Characterization of "single virus" vectors

(A) Transcriptional activity of vectors possessing deletions in the 3'LTR (see Materials and Methods for precise boundaries of deletions). Luciferase activities were determined 48 hours after infection with the different vectors as indicated on the y axis. Enzymatic activities were normalized for the amount of total protein of the samples and the copy number of the luciferase gene in the different cell populations. The activity of the unmodified original SFG Luc vector was set arbitrarily as $100 \%$. (B) Regulation of luciferase activity in NIH3T3 cells infected with "single virus" vectors. NIH3T3 cells were infected with different luciferase vectors as indicated in the "vector" column and split into replica plates. Luciferase activities of the off- (solid bar) and on state (shaded bar) were determined 48 hours after induction of gene expression. The relative transduction efficiency, normalized for proviral copy number, is shown in the "relative transduction efficiency" column. The value of the original SFG Luc vector was arbitrarily set as 1 . The relative induction of gene expression is given in the "relative induction" column. Induction of gene expression, normalization and calculation of the values was done as described in the legend to Figure 2.

quences was accompanied by a progressive decrease in the absolute levels of gene expression that could be achieved in the on state. Of all the vectors, the SFG LucITEl vector, which carried the smallest enhancer deletion, yielded the highest level of luciferase expression (approximately $8 \times$ the level of expression of the parental SFG Luc) and the ability to regulate gene expression over 130-fold. Vectors carrying the other deletions were also highly regulatable (76-100-fold), yet yielded a range of levels of expression in the on state ( 2 to $4 \times$ the levels of SFG Luc).

\section{Regulation of Gene Expression In Vivo}

To explore the feasibility of the use of tet-regulatable retroviral vectors in vivo, we examined the ability of both the two virus and single virus systems to provide for regulated gene expression after the transplantation of murine $\mathrm{C} 2 \mathrm{C} 12$ $(11,12)$ myoblasts transduced by the vectors. For these studies, we employed the human growth hormone (hGH) as a marker gene and vectors which expressed the rtTA gene product. For study of the two virus system, $\mathrm{C} 2 \mathrm{C} 12$ cells were first infected with SFG rtTA and subsequently with SFG tchGH ECT3. For studies of the single virus system, C2C12 cells were transduced by SFG tchGH IRTEl or SFG tchGH IRTE4 (IR designates the use of rtTA rather than tTA). Studies in vitro indicated that in the case of both the two virus and single virus systems, significant regulation of hGH (approx. 34-65 fold) could be achieved through the addition of doxycycline (Fig. 4A). In addition, both vector systems expressed levels of hGH in the on state comparable or greater than that produced by SFG hGH. The lower extent of regulation observed relative to the studies involving luciferase vectors in $\mathrm{NIH}$ $3 T 3$ cells likely relates to a cell type difference in expression of the vectors (20), since experiments involving $\mathrm{C} 2 \mathrm{C} 12$ cells and luciferase vectors also yielded lower extents of regulation (data not shown). 

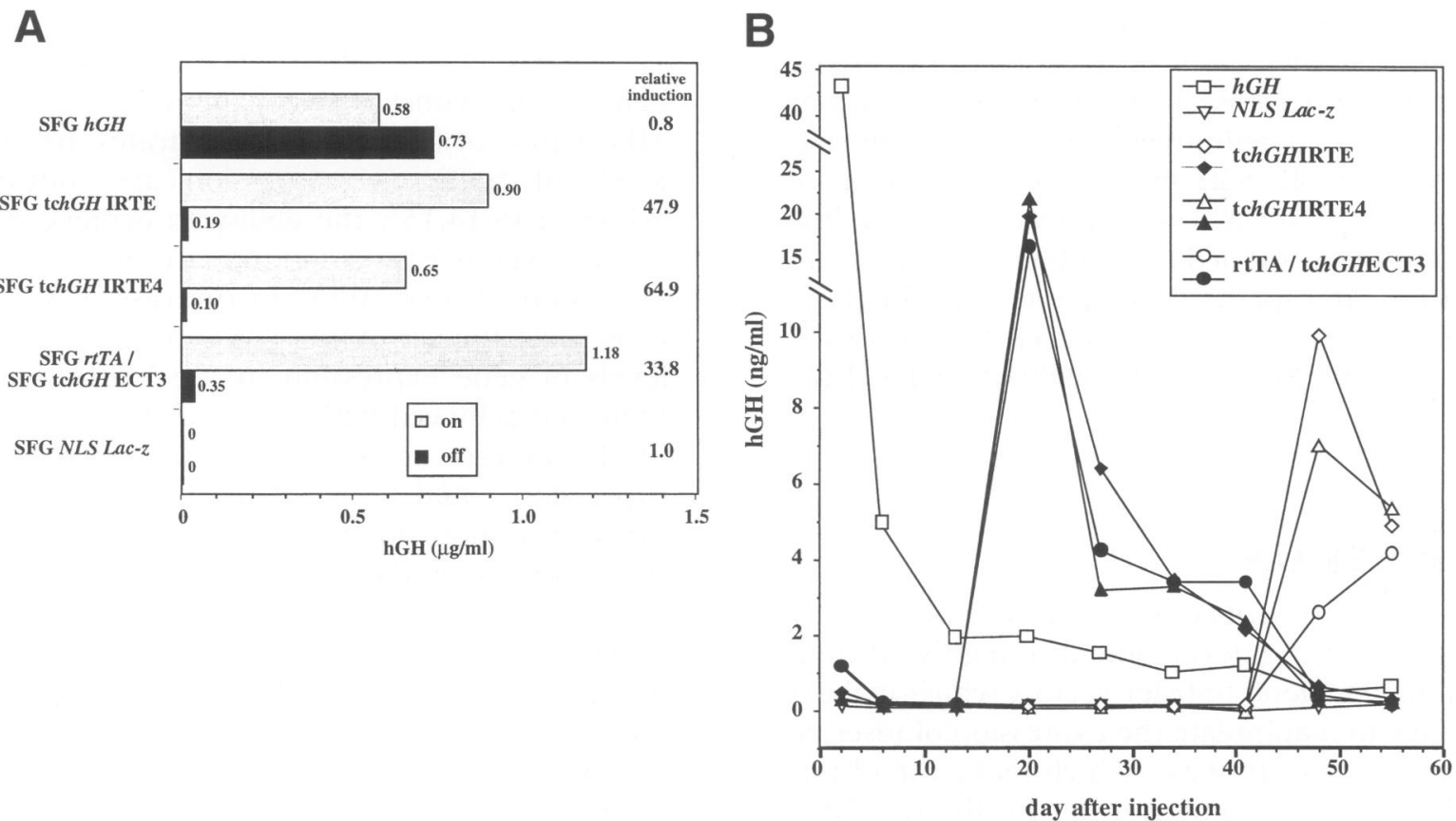

FIG. 4. Regulation of hGH expression in transduced $\mathrm{C} 2 \mathrm{C} 12$ myoblasts in vitro and in vivo

(A) In vitro regulation of hGH expression. $\mathrm{C} 2 \mathrm{C} 12$ myoblasts were infected with different retroviral vectors for constitutive or regulated hGH expression, as indicated on the y axis, as described in Materials and Methods. Two days after infection, $1 \times 10^{6}$ cells were plated into replica plates, one of which contained $1 \mu \mathrm{g} / \mathrm{ml}$ doxycyline in the growth medium. The following day the growth medium was replaced and 24 hours later the supernatant was harvested and levels of secreted hGH in the off- (solid bar) or the on state (shaded bar) were determined by ELISA. (B) In vivo regulation of hGH expression. Groups of 5 to $6 \mathrm{C} 3 \mathrm{H} / \mathrm{HeJ}$ mice received i.m. injections of transduced $\mathrm{C} 2 \mathrm{C} 12$ myoblast populations as described in Materials and Methods. Mice were left untreated up until day 16 after injection when half of the mice of each group were put on doxycycline treatment (dark symbols), while the other half continued to be untreated (light symbols). The treatment of both subgroups was then changed to the opposite on day 46 after injection (e.g. mice treated with doxycycline were taken off the drug and mice not previously treated were given doxycycline). Serum samples of individual mice were taken at the time points indicated in the graph and hGH serum levels were determined by RIA as described in Materials and Methods. Individual values in the graph are the mean of the hGH serum levels of 2 to 6 mice.

In vivo regulation of hGH production by these vectors was examined by injection of transduced $\mathrm{C} 2 \mathrm{C} 12$ myoblast populations into the hind limbs of $\mathrm{C} 3 \mathrm{H} / \mathrm{HeJ}$ mice (21). Gene expression was regulated through the oral administration of doxycycline in the drinking water. At various time points after injection the hGH serum levels in individual mice were determined by RIA. As shown in Figure 4B, mice that received SFG hGH-transduced myoblasts initially expressed high levels of hGH, whether or not doxycycline was administered, yet at subsequent time points showed reduced expression. This decrease in expression most likely reflects the induction of immune responses to hGH. In contrast, mice transplanted with cells transduced by the tetregulatable constructs showed no detectable
hGH in their serum $(<0.15 \mathrm{ng} / \mathrm{ml})$ in the absence of doxycycline, except for low levels detected 2 days post-transplantation. However, when doxycycline was administered either 16 or 46 days after transplantation of cells transduced by the tet-regulatable constructs, large amounts of hGH could be detected when serum from the animals was analyzed 2 days later. At subsequent time points, the levels of hGH decreased, as observed in the case of mice transplanted with SFG hGH transduced cells. While even the initial high levels of hGH gene expression observed in vivo did not appear to directly correlate with the levels of gene expression observed in vitro, it is quite difficult to determine whether this discrepancy reflects the inherent in vivo activity of the tetregulated transcription system employed, or 
factors related to the efficiency of fusion of the transduced myoblasts in vivo. Because of the progressive decrease in hGH expression, it was not possible to definitively demonstrate the ability to shut off hGH expression by removal of antibiotic. Nevertheless, removal of doxycycline 30 days after induction of hGH expression (46 days post-transplantation), a time when hGH expression could readily be detected, was associated with the subsequent inability to detect hGH at later time points.

\section{DISCUSSION}

The studies described above were motivated by the need for the development of highly efficient mammalian gene transfer vectors which make it possible to manipulate the expression of inserted genes. Several strategies which make use of retroviral-mediated gene transfer for the regulated expression of genes have already been already described (22-25). Our current studies extend those previous studies in a number of important ways. In the case of the two virus systems described, we chose to generate separate vectors for the expression of the necessary transactivator gene products and the regulated expression of a desired transgene. Such a strategy makes it possible to express the transactivator without affecting the basal expression of the transgene. We also chose to compare the use of the original tTA transactivator described by Gossen and Bujard (1) to the use of the recently described rtTA (2), since the ability to induce expression with drugs (rather than by removal) has obvious merits, particularly in the context of in vivo delivery of gene products. Our analysis of the expression capabilities of the various constructs tested in the two virus system indicated that gene expression could be regulated 20-60-fold, depending on the constructs, and that similar levels of expression in the induced state were obtained using vectors in which the tet-regulatable transcriptional control sequences were positioned in either the LTR or internal to the proviral transcriptional unit. Only minor differences in the basal levels of expression were observed $(2 \times)$ when a deletion of enhancer, CAAT, and promoter elements in the LTR, rather than a deletion of the enhancer alone, was used. Perhaps the most striking observation made with the two virus constructs was that, in every case, the absolute levels of transgene expression achieved in the induced state was equal to or greater than that achieved with the parental SFG vector. In some cases, 10-fold higher levels than SFG were achieved. In light of our considerable experience with the MFG family of vectors, which indicates that high levels of transgene expression are routinely achieved $(8,14,15)$, the ability to achieve even higher levels of expression than the MFG vectors was unanticipated. Why, in the case of several constructs, the use of the rtTA gene led to higher levels of gene expression in the induced state than that achieved with tTA, remains unclear.

In the case of the single virus system, gene expression could be regulated over a 100-fold range. The most important observation made in the course of studies with the single virus systems was that progressive deletion of transcriptional control sequences in the viral LTR led to proportional decreases in both the basal and induced levels of transgene expression. While the removal of such sequences might be anticipated to reduce the component of basal expression levels due to a low level of LTR-driven transcription, and to perhaps influence expression of the minimal CMV promoter (by reducing enhancement from sequences in the LTR), we do not understand why expression from the regulatable tet/ CMV transcriptional unit would be decreased, rather than increased, by progressive deletion. Although it is possible that the larger deletions affect the stability and/or translational efficiency of the tet/CMV promoted transcripts, studies of gene expression after transfection of SFG-derived constructs carrying those same deletions showed little or no differences in luciferase expression (data not shown). Another potential explanation for the results observed is that the progressive deletions in the LTR reduce the expression of the transactivator gene products to the point they are unable to properly initiate the autostimulation of their expression necessary to provide for the high levels of transgene expression observed with the other constructs. Whatever the explanation, one fortuitous consequence of the phenomenon is that we now have available a family of vectors that lead to distinctly different levels of expression in the induced state. Such vectors may obviate the need to carefully titrate antibiotic concentrations in biological studies in which attaining a specific level of expression is critical. Again, as in the case of the two virus system, we observed, at least with one single virus vector, the ability to achieve levels of gene expression in the induced state higher than those achieved with the parental SFG.

In studies by Blau and co-workers (22) in- 
volving a vector design remarkably similar to that of the single vectors described here, a deletion of both enhancer and promoter sequences in the LTR were employed. While those studies did not provide any information regarding the absolute levels of expression that could be obtained, it is likely, based on our own studies, that the levels of expression that can be achieved in the induced state with their vector would be diminished, relative to that achievable with the parental MFG vector. Although Blau and coworkers also reported the ability to regulate gene expression $600 \times$ with their vector, it should be noted that such a calculation was based on the analysis of a subpopulation of transduced cells isolated by FACS on the basis of low basal expression and high induced expression. Based on the similarity of their constructs and the ones presented here, it is likely that the basal levels of expression of the different constructs would be comparable.

Lastly, the experiments involving the transplantation of cells transduced by the regulatable vectors are encouraging for the ultimate use of such vectors in vivo. In conjunction with the recent development of packaging cell lines which make it possible to generate high titers of viral pseudotypes which can be highly concentrated $(26,27)$, and the demonstration of the ability of lentivirus vectors to efficiently transduce at least some quiescent cells (28), the work reported here suggests that obtaining the regulated expression of genes via the direct in vivo delivery of retroviral vectors will ultimately prove to be feasible.

\section{ACKNOWLEDGMENTS}

We thank $\mathrm{H}$. Bujard for providing the plasmids pUHC13-3, pUHD15-1, and pUHG17-1. Bosc23 cells were a gift of W. Pear. The work was supported by NIH grant UOlAl26463. D.L. was supported by a grant from the Deutsches Krebsforschungszentrum.

\section{REFERENCES}

1. Gossen M, Bujard H. (1992) Tight control of gene expression in mammalian cells by tetracycline-responsive promoters. Proc. Natl. Acad. Sci. USA 89 12: 5547-5551.

2. Gossen M, Freundlieb S, Bender G, Müller G, Hillen W, Bujard H. (1995) Transcrip- tional Activation by Tetracyclines in Mammalian Cells. Science 268, 23. June 1995: 1766-1769.

3. Rivera V, Clackson T, Natesan S, Pollock R, Amara J, Keenan T, Magari S, Phillips T, Courage N, Cerasoli FJ, Holt D, Gilman M. (1996) A humanized system for pharmacologic control of gene expression [see comments]. Nat. Med. 2, 9: 1028-1032.

4. No D, Yao T, Evans R. (1996) Ecdysoneinducible gene expression in mammalian cells and transgenic mice. Proc. Natl. Acad. Sci. USA 93, 8: 3346-3351.

5. Hillen W, W.A. (1989) TetR-tetO Interaction. In Protein-Nucleic Acid Interaction, vol. 10. H.U. Saenger W, editor. Macmillan Press, London. 143-162.

6. Furth PA, St Onge L, Boger H, Gruss P, Gossen M, Kistner A, Bujard H, Hennighausen L. (1994) Temporal control of gene expression in transgenic mice by a tetracycline-responsive promoter. Proc. Natl. Acad. Sci. U.S.A. 91, 20: $9302-9306$.

7. Gossen M, Bonin A, Freundlieb S, Bujard H. (1994) Inducible gene expression systems for higher eukaryotic cells. Curr. Opin. Biotechnol. 5, 5: 516-520.

8. Bueler H, Mulligan RC. (1996) Induction of antigen-specific tumor immunity by genetic and cellular vaccines against MAGE: enhanced tumor protection by coexpression of granulocyte-macrophage colony-stimulating factor and B7-1. Mol. Med. 2: 545-555.

9. Dranoff G, Jaffee E, Lazenby A, Golumbek P, Levitsky H, Brose K, Jackson V, Hamada H, Pardoll D, Mulligan RC. (1993) Vaccination with irradiated tumor cells engineered to secrete murine granulocyte-macrophage colony-stimulating factor stimulates potent, specific, and long-lasting anti-tumor immunity. Proc. Natl. Acad. Sci. U.S.A. 90, 8: 3539-3543.

10. Riviere I, Brose K, Mulligan RC. (1995) Effects of retroviral vector design on expression of human adenosine deaminase in murine bone marrow transplant recipients engrafted with genetically modified cells. Proc. Natl. Acad. Sci. U.S.A. 92, 15: 6733-6737.

11. Cone RD, Weber-Benarous A, Baorto D, Mulligan RC. (1987) Regulated expression of a complete human beta-globin gene encoded by a transmissible retrovirus vector. Mol. Cell. Biol. 7, 2: 887-897.

12. Yu SF, von Ruden T, Kantoff PW, Garber C, Seiberg M, Ruther U, Anderson WF, Wagner EF, Gilboa E. (1986) Self-inactivating retro- 
viral vectors designed for transfer of whole genes into mammalian cells. Proc. Natl. Acad. Sci. U.S.A. 83, 10: 3194-3198.

13. Yee JK, Moores JC, Jolly DJ, Wolff JA, Respess JG, Friedmann T. (1987) Gene expression from transcriptionally disabled retroviral vectors. Proc. Natl. Acad. Sci. U.S.A. 84, 15: 5197-5201.

14. Jang SK, Wimmer E. (1990) Cap-independent translation of encephalomyocarditis virus RNA: structural elements of the internal ribosomal entry site and involvement of a cellular 57-kD RNA-binding protein. Genes. Dev. 4, 9: 1560-1572.

15. Pear WS, Nolan GP, Scott ML, Baltimore D. (1993) Production of high-titer helper-free retroviruses by transient transfection. Proc. Natl. Acad. Sci. U.S.A. 90, 18: 8392-8396.

16. Yaffe D, Saxel O. (1977) Serial passaging and differentiation of myogenic cells isolated from dystrophic mouse muscle. Nature 270: 725-727.

17. Blau HM, Chiu CP, Webster C. (1983) Cytoplasmic Activation of Human Nuclear Genes in Stable Heterocaryons. Cell 32: 1171-1180.

18. Ackland-Berglund CE, Leib DA. (1995) Efficacy of tetracycline-controlled gene expression is influenced by cell type. Biotechniques 18, 2: 196-200.

19. Dhawan J, Pan LC, Pavlath GK, Travis MA, Lanctot AM, Blau HM. (1991) Systemic delivery of human growth hormone by injection of genetically engineered myoblasts [see comments]. Science 254, 5037: 1509-1512.

20. Hofmann A, Nolan GP, Blau HM. (1996) Rapid retroviral delivery of tetracycline-inducible genes in a single autoregulatory cassette. Proc. Natl. Acad. Sci. U.S.A. 93, May 1996: 5185-5190.

21. Hwang J, Scuric Z, Anderson W. (1996)
Novel retroviral vector transferring a suicide gene and a selectable marker gene with enhanced gene expression by using a tetracycline-responsive expression system. J. Virol. 70, 11: 8138-8141.

22. Iida $A$, Chen $S-T$, Friedmann $T$, Yee J-K. (1996) Inducible Gene Expression by Retrovirus-Mediated Transfer of a Modified Tetracycline-Regulated System. J. Virol. 70, 9: 6054-6059.

23. Paulus W, Baur I, Boyce FM, Breakefield XO, Reeves SA. (1996) Self-contained, tetracycline-regulated retroviral vector system for gene delivery to mammalian cells. $J$. Virol. 70, 1: 62-67.

24. Ory D, Neugeboren B, Mulligan R. (1996) A stable human-derived packaging cell line for production of high titer retrovirus/vesicular stomatitis virus G pseudotypes. Proc. Natl. Acad. Sci. U.S.A. 93, 21: 11400-11406.

25. Yang $Y$, Vanin $E$, Whitt $M$, Fornerod $M$, Zwart R, Schneiderman R, Grosveld G, Nienhuis A. (1995) Inducible, high-level production of infectious murine leukemia retroviral vector particles pseudotyped with vesicular stomatitis virus $\mathrm{G}$ envelope protein. Hum. Gene. Ther. 6, 9: 1203-1213.

26. Naldini L, Blomer U, Gallay P, Ory D, Mulligan $\mathrm{R}$, Gage FH, Verma IM, Trono D. (1996) In vivo gene delivery and stable transduction of nondividing cells by a lentiviral vector. Science 272, 5259: 263-267.

27. Martial JA, Hallewell RA, Baxter JD, Goodman HM. (1979) Human growth hormone: complementary DNA cloning and expression in bacteria. Science 205, 4406: 602-607.

28. Ferry N, Duplessis O, Houssin D, Danos O, Heard JM. (1991) Retroviral-mediated gene transfer into hepatocytes in vivo. Proc. Natl. Acad. Sci. U.S.A. 88, 19: 8377-8381.

Communicated by C. Weissmann. Accepted May 15, 1997. 